\title{
Asymptotic Structure of the Seismic Radiation from an Explosive Column
}

\author{
Marco Rosales-Vera $\mathbb{D}^{1},{ }^{1}$ Iván Piñeyro, ${ }^{1}$ and Gastón Pinilla ${ }^{2}$ \\ ${ }^{1}$ CODELCOTECH, Los Jardines 279, Santiago, Chile \\ ${ }^{2}$ CODELCO CHILE, Gerencia Corporativa de Innovación y Tecnología, Huérfanos 1270, Santiago, Chile \\ Correspondence should be addressed to Marco Rosales-Vera; mrosa001@codelcotech.cl
}

Received 27 September 2017; Revised 13 February 2018; Accepted 18 February 2018; Published 29 March 2018

Academic Editor: Emil Manoach

Copyright (c) 2018 Marco Rosales-Vera et al. This is an open access article distributed under the Creative Commons Attribution License, which permits unrestricted use, distribution, and reproduction in any medium, provided the original work is properly cited.

We study the structure of the seismic radiation in the far field produced by an explosive column. Using an asymptotic solution for the far field of vibration (Heelan's solution), we find analytical expressions to the peak particle velocity (PPV) diagrams. These results are extended to the case of a charge with finite velocity of detonation.

\section{Introduction}

The radiation of elastic waves produced by a cylindrical charge in a borehole has important applications in seismic exploration, mining geophysics, and blast vibration prediction. Today it is a well-known fact that, in the blasting engineering, the curves of blasting vibration in the near field and the far field show that vibration amplitudes induced by $P$ wave and $S$ wave are generally in the same magnitude, so the effect of $S$ wave could not be ignored.

A pioneering theoretical work is that developed by Heelan (1953) $[1,2]$, where he study the radiation from a cylindrical source of finite length, the walls of which are subjected to symmetric lateral and tangential stresses. His papers were credited with demonstrating the generation of shear waves by artificial sources in a borehole; at the time this was a fundamentally surprising result. Subsequently, several authors have been making improvements to the results of Heelan, principally by Jordan [3], Hazebroek [4], and AboZena [5]. One of the main advantages of the Heelan solution is its algebraic simplicity and clear physical interpretation, which allows easy implementation in more complex models.

An exact analytical solution of the problem solved by Heelan [2] was developed by Meredith [6]; Meredith et al. [7] and Meredith et al. [8] analyze the exact full-field solution for the axisymmetric wave radiation caused by any time-varying pressure load source acting over a finite length of a borehole. Tubman [9] and Tubman et al. [10] derive a general expression for the dispersion relations and the impulse response of a radially layered borehole.

Blair and Minchinton [11] analyze the near field of vibration from an explosive column; Blair [12] makes a comparison between Heelan solution and exact solutions for seismic radiation from a short cylindrical charge. Analytical solutions for describing Mach waves produced by infinitely long explosive columns were studied by Rossmanith and Kouzniak [13]. Blair [14] studied the blast vibration dependence on charge length, velocity of detonation, and layered media. Triviño et al. [15] studied seismic radiation patterns from cylindrical explosive charges by analytical and combined finite-discrete element methods. Kumar et al. [16] analyze the blast-induced ground vibration equations for rocks using mechanical and geological properties. Lee and Balch [17] generalize the results of Heelan to a fluid-filled borehole. The interaction between two adjacent blast holes has been studied by Yi et al. [18]. Triviño et al. [19] have studied the seismic waveforms from explosive sources located in boreholes and initiated in different directions. Using the Heelan results, Chen et al. [20] have studied the influence of millisecond time, charge length, and detonation velocity on blasting vibration. 


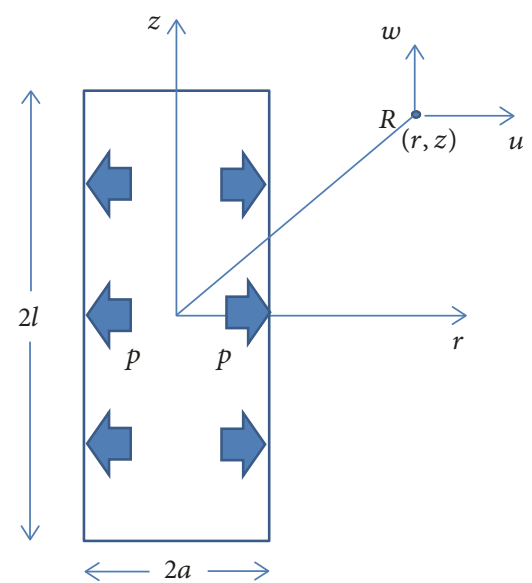

FIGURE 1: Schematic of a cylindrical charge.

In this work we study the structure of the seismic radiation in the far field produced by an explosive column. Using an asymptotic solution for the far field of vibration (Heelan's solution), we find analytical expressions to the peak particle velocity (PPV) diagrams. The analysis is extended to the case of a charge with finite velocity of detonation, obtaining a first correction to the Heelan's solution in the case of finite VOD. The results are compared with those existing in the literature.

\section{Mathematical Model}

2.1. Short Cylindrical Charge (Heelan's Solution). Consider a cylindrical explosive charge length $2 l$ and radius $a$, centered at the origin of coordinates and embedded in an infinite elastic medium (Figure 1). The vertical walls of this cylinder are subjected to stresses which are symmetric about the vertical axis, uniform in the sense that they have the same instantaneous value at all points, and finite in time duration.

It has been shown (Heelan 1953) that for a pressure transient acting on the walls of a short section of an infinitely long cylindrical hole of radius $a$, the far field radial and tangential displacements are

$$
\begin{aligned}
& u_{R}(R, \phi, t) \\
& =\frac{l a^{2}}{2 \mu c_{p} R}\left(1-2\left(\frac{c_{s}}{c_{p}}\right)^{2} \cos ^{2}(\phi)\right) \frac{d}{d t}\left\{p\left(t-\frac{R}{c_{p}}\right)\right\} \\
& u_{\phi}(R, \phi, t)=\frac{l a^{2}}{2 \mu c_{s} R} \sin (2 \phi) \frac{d}{d t}\left\{p\left(t-\frac{R}{c_{s}}\right)\right\} .
\end{aligned}
$$

To simplify the notation we will define the coefficients $F_{1}(\phi)$ and $F_{2}(\phi)$ :

$$
\begin{aligned}
& F_{1}(\phi)=\frac{l a^{2}}{2 \mu c_{p}}\left(1-2\left(\frac{c_{s}}{c_{p}}\right)^{2} \cos ^{2}(\phi)\right) \\
& F_{2}(\phi)=\frac{l a^{2}}{2 \mu c_{s}} \sin (2 \phi),
\end{aligned}
$$

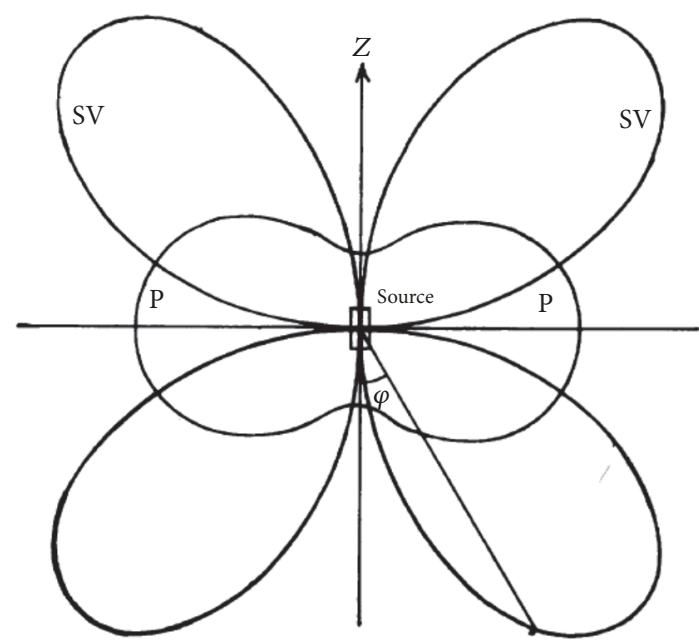

Figure 2: Angular distribution of $\mathrm{P}$ and $\mathrm{S}$ waves.

where $2 l$ is the total length of the loaded section of the cylinder, $\phi$ is the polar angle measured from the cylinder's axis, $R$ is the distance to the observer, $c_{s}=\sqrt{\mu / \rho}$ and $c_{p}=$ $\sqrt{(\lambda+2 \mu) / \rho}$ are the shear and compressional wave speeds in the medium, and $\mu$ and $\rho$ are the rock's rigidity and density, respectively. In this analysis, the source pressure function is based on the experimental data of Grady et al. [21] and Larson [22] and is given by

$$
P(t)=P_{\mathrm{VN}}\left(\frac{e \gamma}{n}\right)^{n} t^{n} e^{-\gamma t} H(t),
$$

where " $e$ " is the Euler's constant, $P_{\mathrm{VN}}$ is the von Neumann borehole pressure at the detonation front produced by the explosive, $H(t)$ is the Heaviside unit step function, $n$ is an integer, and $\gamma$ is a pressure decay parameter. The values of the parameter $n$ are in the range of 3 to 9 .

Equations (1) show that, in the far field, radial and tangential displacements travel as waves in the solid ( $\mathrm{S}$ wave and $\mathrm{P}$ wave), with speeds $c_{s}$ and $c_{p}$, respectively, where $c_{p}>c_{s}$. Another important feature of the solution of Heelan is the angular variation of the amplitude of the radiated $\mathrm{P}$ and $\mathrm{S}$ waves, where the displacement induced by shear wave could be larger than that caused by $\mathrm{P}$ wave. Figure 2 shows the angular distribution of $\mathrm{P}$ and $\mathrm{S}$ waves.

You can see that there are regions where the $\mathrm{P}$ waves dominate over the $S$ waves and vice versa. Clearly the $S$ waves predominate around $\phi_{n}=(2 n+1)(\pi / 4)$, with $n=0,1,2,3$. The $\mathrm{P}$ waves predominate around $\phi_{n}=n(\pi / 2)$. The region where the $\mathrm{P}$ waves predominate over $S$ waves can be estimated from the relationship $F_{1}(\phi) \geq F_{2}(\phi)$; then

$$
\left(1-2\left(\frac{c_{s}}{c_{p}}\right)^{2} \cos ^{2}\left(\phi^{*}\right)\right) \geq \frac{c_{p}}{c_{s}} \sin \left(2 \phi^{*}\right) .
$$

This equation leads to the following expression:

$$
\cos ^{2}\left(\phi^{*}\right)=\frac{1}{2} \frac{1}{\left(1+\bar{\lambda}^{6}\right)}\left(1+\bar{\lambda}^{4} \pm \sqrt{1-\bar{\lambda}^{2}+2 \bar{\lambda}^{4}}\right),
$$


where $\bar{\lambda}=c_{s} / c_{p}<1$. The angular widths for windows radiation $\mathrm{P}$ waves are given by

$$
\begin{aligned}
& \Delta \phi_{1}=2 \cos ^{-1}\left(A_{+}\right) \\
& \Delta \phi_{2}=\pi-2 \cos ^{-1}\left(A_{-}\right),
\end{aligned}
$$

where the parameters $A_{+,-}$are given by

$$
A_{+,-}=\sqrt{\frac{1}{2} \frac{1}{\left(1+\bar{\lambda}^{6}\right)}\left(1+\bar{\lambda}^{4} \pm \sqrt{1-\bar{\lambda}^{2}+2 \bar{\lambda}^{4}}\right)} .
$$

Figure 3 shows the angular width for the $\mathrm{P}$ wave radiation as a function of the parameter $\bar{\lambda}$; it can be seen that the angular aperture for the radiation field of $\mathrm{P}$ waves in general is less than that of $S$ waves, which shows the importance of $S$ waves in the fracture process material by detonating cylindrical explosive charges.

2.2. Particle Velocity. The velocity time histories $v_{R}(r, z, t)$ and $v_{\phi}(r, z, t)$ predicted by the Heelan solution for the pressure function equation are

$$
\begin{aligned}
& v_{R}(R, \phi, t)=\frac{l a^{2}}{2 \mu c_{p} R}\left(1-2\left(\frac{c_{s}}{c_{p}}\right)^{2}\right. \\
& \left.\cdot \cos ^{2}(\phi)\right) \frac{d^{2}}{d t^{2}}\left\{p\left(t-\frac{R}{c_{p}}\right)\right\} \\
& v_{\phi}(R, \phi, t)=\frac{l a^{2}}{2 \mu c_{s} R} \sin (2 \phi) \frac{d^{2}}{d t^{2}}\left\{p\left(t-\frac{R}{c_{s}}\right)\right\} .
\end{aligned}
$$

The particle velocity is defined as $|\vec{u}|=\sqrt{v_{R}^{2}+v_{\phi}^{2}}$,

$$
|\vec{u}|=\sqrt{\left(\frac{F_{1}(\phi)}{R} \frac{d^{2}}{d t^{2}}\left\{p\left(t-\frac{R}{c_{p}}\right)\right\}\right)^{2}+\left(\frac{F_{2}(\phi)}{R} \frac{d^{2}}{d t^{2}}\left\{p\left(t-\frac{R}{c_{s}}\right)\right\}\right)^{2}}
$$

In general, (9) is a complex function of the coordinates and time, but we will see that it may be susceptible to considerable simplifications given the angular structure discussed above.

Besides, $\mathrm{P}$ wave and $\mathrm{S}$ wave produced by blasting will separate gradually because of their velocity differences when the distance from measured point to blasting source increases. Another important aspect is that the width of the wave fronts are given approximately by $\delta_{p} \sim\left(c_{p} / \gamma\right)$ and $\delta_{s} \sim\left(c_{s} / \gamma\right)$ for $\mathrm{P}$ wave and $\mathrm{S}$ wave, respectively. Thus, the Heelan model shows that the $\mathrm{P}$ wave and the $\mathrm{S}$ wave do not overlap in time.

Figure 4 shows the advancing wave fronts as a function of time and the spatial coordinates. The values of the parameters used are $c_{p}=5000 \mathrm{~m} / \mathrm{s}, c_{s}=3000 \mathrm{~m} / \mathrm{s}, a=2 l=0.1 \mathrm{~m}$, and three different values of the parameter $\gamma$.

It can be seen that as the value of the parameter $\gamma$ increases, the width of the wave fronts decrease, making more evident uncoupling between the $\mathrm{P}$ and $\mathrm{S}$ waves. However, the angular distribution of the radiation remains intact following the distribution given by (4). Another important aspect is that the regions near the axis of the explosive charge, vibration induced by the explosive charge, are very low; this feature is also explained by (4), where the angular opening for waves in this region is very small.

2.3. The Peak Particle Velocity. The peak particle velocity is given by

$$
|\vec{u}|_{\mathrm{PP}}=\max \left(\sqrt{\left(\frac{F_{1}(\phi)}{R} \frac{d^{2}}{d t^{2}}\left\{p\left(t-\frac{R}{c_{p}}\right)\right\}\right)^{2}+\left(\frac{F_{2}(\phi)}{R} \frac{d^{2}}{d t^{2}}\left\{p\left(t-\frac{R}{c_{s}}\right)\right\}\right)^{2}}\right) .
$$

As demonstrated above, the $\mathrm{P}$ wave and the $\mathrm{S}$ wave do not overlap in time. Thus, the peak particle velocity can be expressed approximately as follows:

$$
\begin{aligned}
|\vec{u}|_{\mathrm{PP}} \approx \max \left\{\frac{F_{1}(\phi)}{R} \frac{d^{2}}{d t^{2}}\left\{p\left(t-\frac{R}{c_{p}}\right)\right\}, \frac{F_{2}(\phi)}{R}\right. \\
\left.\cdot \frac{d^{2}}{d t^{2}}\left\{p\left(t-\frac{R}{c_{s}}\right)\right\}\right\} .
\end{aligned}
$$

Note that the maximum value of peak particle velocity in time depends only on the maximum value of the function $\left(d^{2} / d t^{2}\right)\left\{p\left(t-R / c_{p, s}\right)\right\}$; that is,

$$
\begin{aligned}
& \max \left\{\frac{d^{2}}{d t^{2}}\left\{p\left(t-\frac{R}{c_{p, s}}\right)\right\}\right\}=\frac{\gamma^{2} a P_{\mathrm{VN}} l}{2 \mu c_{p, s}}\left(\frac{e}{n}\right)^{n} G_{n} \\
& =\alpha_{n},
\end{aligned}
$$

where $G_{n}$ is a numerical value that depends on $n$, for $n=$ $4, G_{n}=1.85$. 


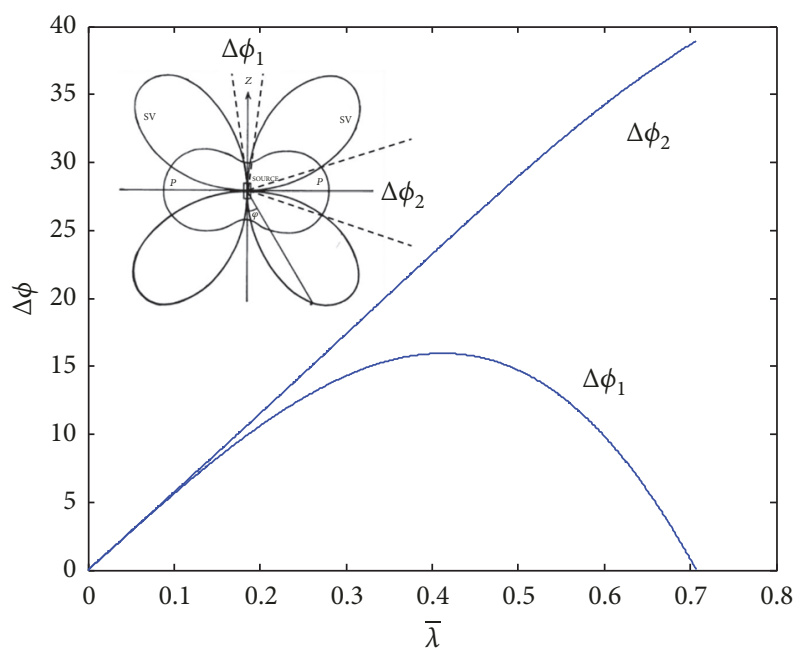

Figure 3: Angular width for the $\mathrm{P}$ wave radiation as a function of the parameter $\bar{\lambda}$.

Finally, taking into account the above considerations, the following approximate expression for the PPV is obtained:

$$
|\vec{u}|_{\mathrm{PP}} \approx \begin{cases}\alpha_{n} \frac{F_{1}(\phi)}{R} \quad & 0 \leq \phi \leq \frac{1}{2} \Delta \phi_{1} \\ & \frac{1}{2} \pi-\frac{1}{2} \Delta \phi_{2} \leq \phi \leq \frac{1}{2} \pi-\frac{1}{2} \Delta \phi_{2} \\ & \pi-\frac{1}{2} \Delta \phi_{1} \leq \phi \leq \pi+\frac{1}{2} \Delta \phi_{1} \\ \alpha_{n} \frac{F_{2}(\phi)}{R} \quad & \frac{1}{2} \Delta \phi_{1} \leq \phi \leq \frac{1}{2} \pi-\frac{1}{2} \Delta \phi_{2} \\ & \frac{1}{2} \pi+\frac{1}{2} \Delta \phi_{2} \leq \phi \leq \pi-\frac{1}{2} \Delta \phi_{1} .\end{cases}
$$

Figure 5 shows the PPV diagrams numerically obtained from (10) (continuous line) compared with the analytic solution (13) (dashed line). You can see that the analytical solution predicts quite well the numerical results, showing that this analytical solution allows a useful tool for simple and rapid assessments under field conditions.

\section{Large Cylindrical Charge $a / L \ll 1$}

In the case when the length $(L)$ of the cylindrical charge is not small, the angular distribution of the velocity field is very different from the case of a charge of small length. Mainly because when $a / L \ll 1$, the detonation of the explosive cannot be considered instant and it becomes necessary to consider the finite velocity of detonation VOD. A large cylindrical charge can be modeled as a superposition of an array of $n$ Heelan's elements considering a delay time for the detonation of each element (Figure 6). The length of each element is defined as $\Delta L=L / 2 n$.
3.1. Particle Velocity. The field generated by the superposition of the charges is given by

$$
\begin{aligned}
& u_{R}(r, z, t)=\sum_{i=1}^{n} u_{R}^{(i)}(r, z, t) \\
& u_{\phi}(r, z, t)=\sum_{i=1}^{n} u_{\phi}^{(i)}(r, z, t),
\end{aligned}
$$

where the field generated by the $i$ th charge located in position $z_{i}$ is given by

$$
\begin{aligned}
& u_{R}^{(i)}(r, z, t)=\frac{F_{1}\left(\phi_{i}\right)}{R_{i}} \frac{d^{2}}{d t^{2}}\left\{p\left(t-\frac{R_{i}}{c_{p}}-\frac{z_{i}}{c_{d}}\right)\right\} \\
& u_{\phi}^{(i)}(r, z, t)=\frac{F_{2}\left(\phi_{i}\right)}{R_{i}} \frac{d^{2}}{d t^{2}}\left\{p\left(t-\frac{R_{i}}{c_{s}}-\frac{z_{i}}{c_{d}}\right)\right\}
\end{aligned}
$$

with

$$
\begin{aligned}
\cos \phi_{i} & =-\frac{z-z_{i}}{R_{i}} \\
\sin \phi_{i} & =\frac{r}{R_{i}} \\
R_{i} & =\sqrt{r^{2}+\left(z-z_{i}\right)^{2}} \\
F_{1}\left(\phi_{i}\right) & =\frac{a^{2} \Delta L}{2 \mu c_{p}}\left(1-2\left(\frac{c_{s}}{c_{p}}\right)^{2} \cos ^{2}\left(\phi_{i}\right)\right) \\
F_{2}\left(\phi_{i}\right) & =\frac{a^{2} \Delta L}{2 \mu c_{s}} \sin \left(2 \phi_{i}\right) .
\end{aligned}
$$

Figure 7 shows the advancing wave fronts as a function of time and the spatial coordinates. The values of the parameters used are $c_{p}=5000 \mathrm{~m} / \mathrm{s}, c_{s}=3000 \mathrm{~m} / \mathrm{s}, L=6 \mathrm{~m}$, and three different values of the parameter $\gamma$. From Figure 7 it can be seen that (14) reproduces well the fronts of $\mathrm{P}$ and $\mathrm{S}$ waves, where cones formed by these fronts are appreciated. For smaller times $\left(t<L / c_{d}\right)$, represented fronts coincide quite well with those found by Rossmanith and Kouzniak [13].

3.2. The Peak Particle Velocity. In this case, the peak particle velocity is given by

$$
\begin{aligned}
& |\vec{u}|_{\mathrm{PP}} \\
& =\max \left(\sqrt{\left(\sum_{i=1}^{n} u_{R}^{(i)}(r, z, t)\right)^{2}+\left(\sum_{i=1}^{n} u_{\phi}^{(i)}(r, z, t)\right)^{2}}\right) .
\end{aligned}
$$

Figure 8 shows the PPV diagrams numerically obtained from (17). The values of the parameters used are $c_{p}=5000 \mathrm{~m} / \mathrm{s}$ and $c_{s}=3000 \mathrm{~m} / \mathrm{s}$. To analyze the effect of the charge length $L$ and attenuation coefficient on the PPV diagrams, three different values for these parameters were considered. The results show that, for moderate charge lengths $(L \sim 1 \mathrm{~m})$ and moderates values of $\gamma$, the PPV diagrams (Figure $8(\mathrm{a})$ ) are very similar 

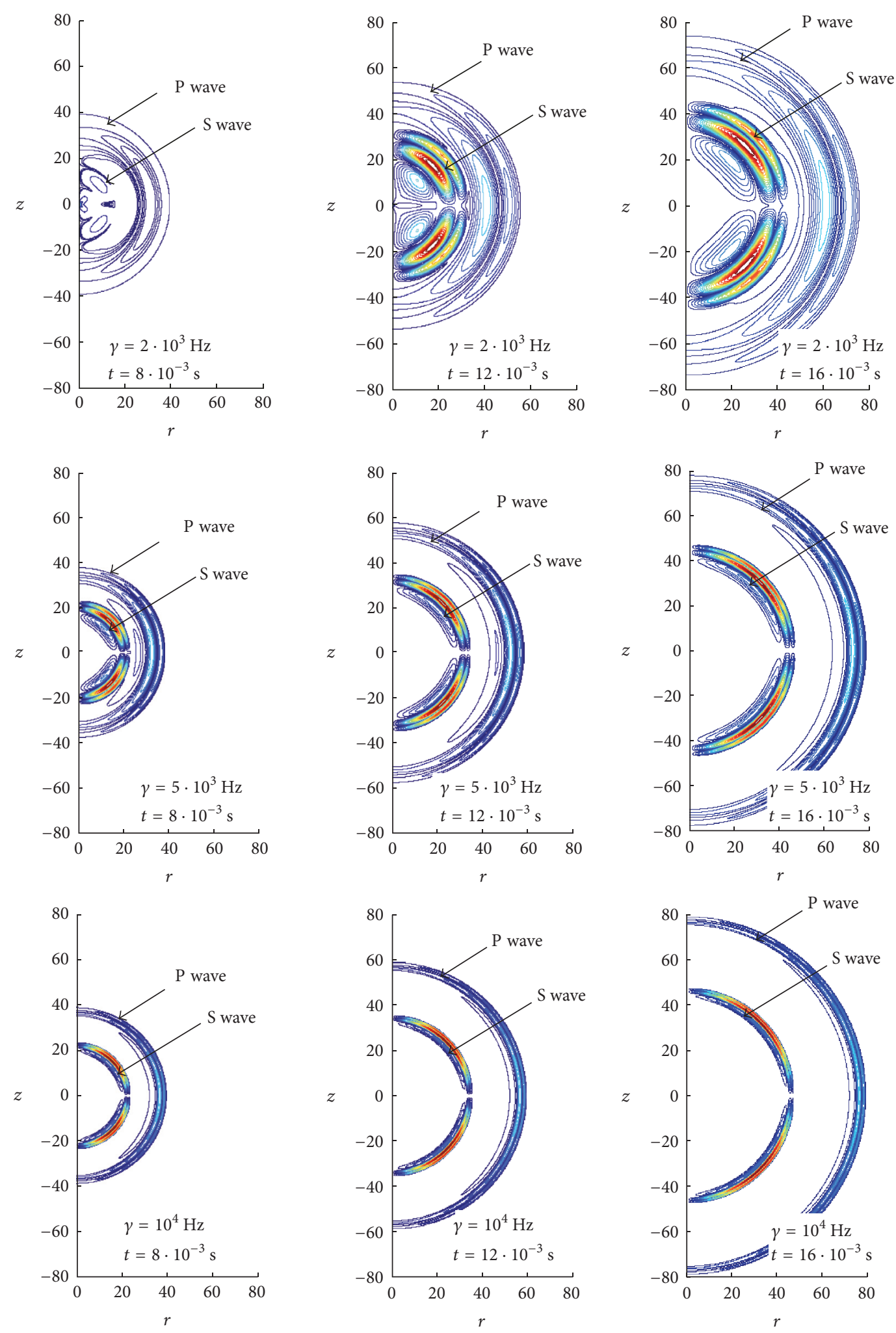

Figure 4: $\mathrm{P}$ and $\mathrm{S}$ wave radiation patterns for source in a borehole wave fronts as a function of time and the spatial coordinates. The values of the parameters used are $c_{p}=5000 \mathrm{~m} / \mathrm{s}, c_{s}=3000 \mathrm{~m} / \mathrm{s}, a=2 l=0.1 \mathrm{~m}$, and three different values of the parameter $\gamma$. 

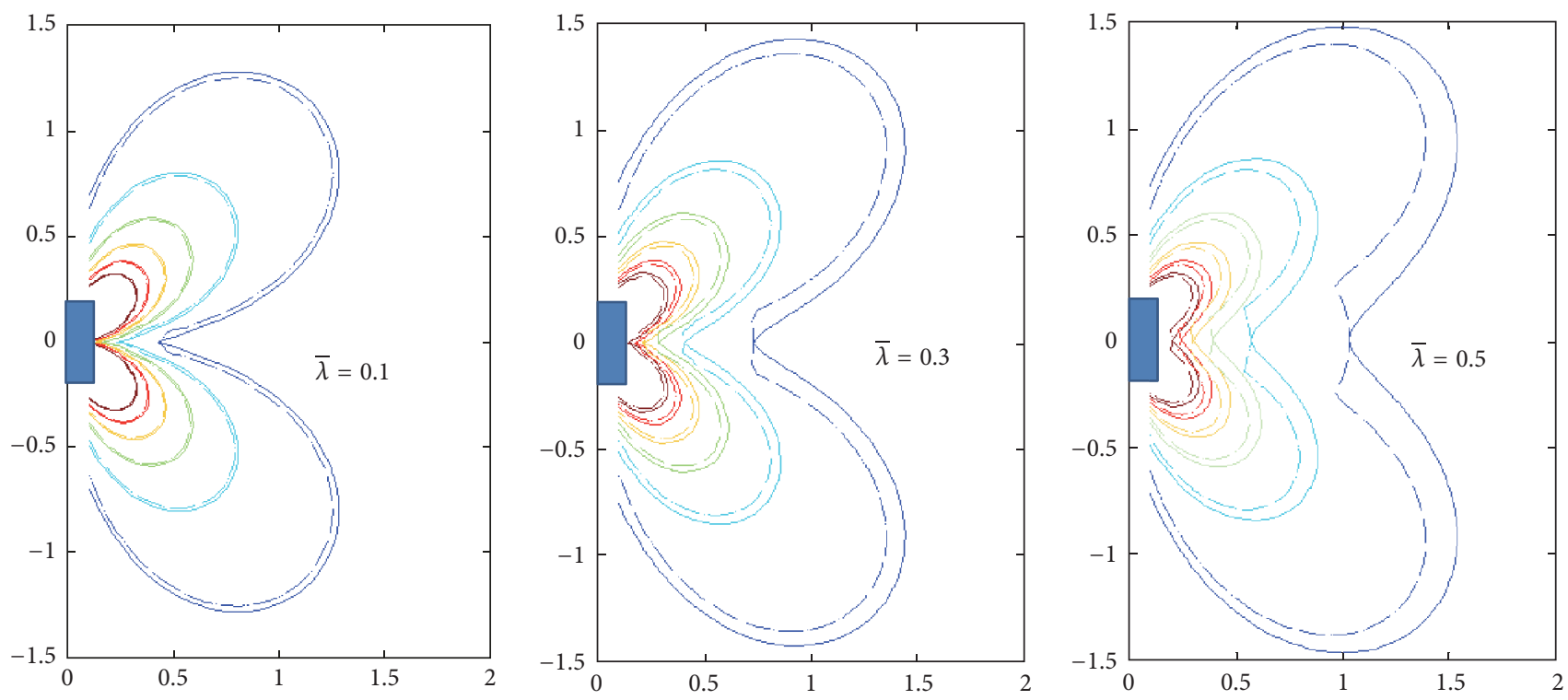

FIgURE 5: PPV diagrams for different values of $\bar{\lambda}=c_{s} / c_{p}$.

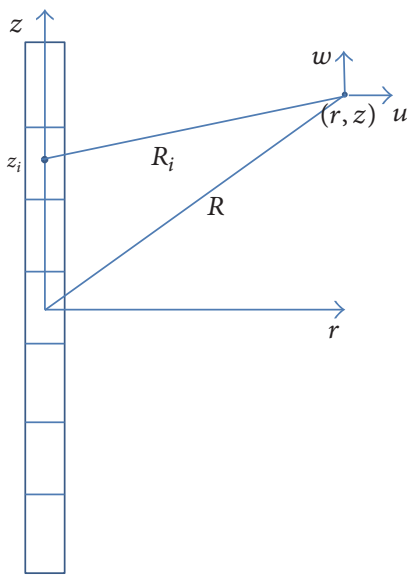

FIGURE 6: Large cylindrical charge modeled as a superposition of an array of elements of Heelan.

to those delivered by Heelan solution for a charge of small length. As gamma increases (at constant $L$ ), the symmetry of the PPV diagrams with respect to the plane $z=0$ is broken, showing a higher intensity of vibration in opposite to the initiation of detonation regions (Figures 8 (b)-8(c)). The same effect is found if $\gamma$ is kept fixed and the value of the length of the load increases (Figures $8(\mathrm{e})-8(\mathrm{~h})$ ), which shows that there is a relationship between $\gamma$ and $L$ governing structure diagrams PPV.

3.3. Approximate Analytical Solution. To find an analytical solution of the field of vibration, we can take the following approach in the far field:

$$
\begin{aligned}
R_{i} & =R-z_{i} \cos \phi_{i} \\
\phi_{i} & =\phi .
\end{aligned}
$$

In the case $n=0$, the field generated by the $i$ th charge located in position $z_{i}$ is given by

$$
\begin{aligned}
& p\left(t-\frac{R_{i}}{c_{p}}-\frac{z_{i}}{c_{d}}\right) \\
& =p_{0} e^{-\gamma\left(t-R_{i} / c_{p}-z_{i} / c_{d}\right)} H\left(t-\frac{R_{i}}{c_{p}}-\frac{z_{i}}{c_{d}}\right)
\end{aligned}
$$

Introducing ((18)-(19)) into (14), it is not difficult to show that the fields of vibration can be approximated by

$$
\begin{aligned}
& \sum_{i=1}^{n} u_{R}^{(i)}(r, z, t)=p_{0} \gamma^{2} \frac{F_{1}(\phi)}{R} \\
& \cdot e^{-\gamma\left(t-R / c_{p}+z_{n}\left(\cos \phi / c_{p}-1 / c_{d}\right)\right)} \sum_{i=0}^{n} e^{\gamma i \Delta L\left(\cos \phi / c_{p}-1 / c_{d}\right)} \\
& \sum_{i=1}^{n} u_{\phi}^{(i)}(r, z, t)=p_{0} \gamma^{2} \frac{F_{2}(\phi)}{R} \\
& \cdot e^{-\gamma\left(t-R / c_{s}+z_{n}\left(\cos \phi / c_{s}-1 / c_{d}\right)\right)} \sum_{i=0}^{n} e^{\gamma i \Delta L\left(\cos \phi / c_{s}-1 / c_{d}\right)} .
\end{aligned}
$$

Finally, the following expressions for the velocity fields are found:

$$
\begin{gathered}
u_{R}(r, z, t) \sim p_{0} \gamma^{2} \frac{F_{1}(\phi)}{R} e^{-\gamma\left(t-R / c_{p}+L\left(\cos \phi / c_{p}-1 / c_{d}\right)\right)} \\
\cdot \frac{1-e^{\gamma L\left(\cos \phi / c_{p}-1 / c_{d}\right)}}{1-e^{\gamma \Delta L\left(\cos \phi / c_{p}-1 / c_{d}\right)}}
\end{gathered}
$$



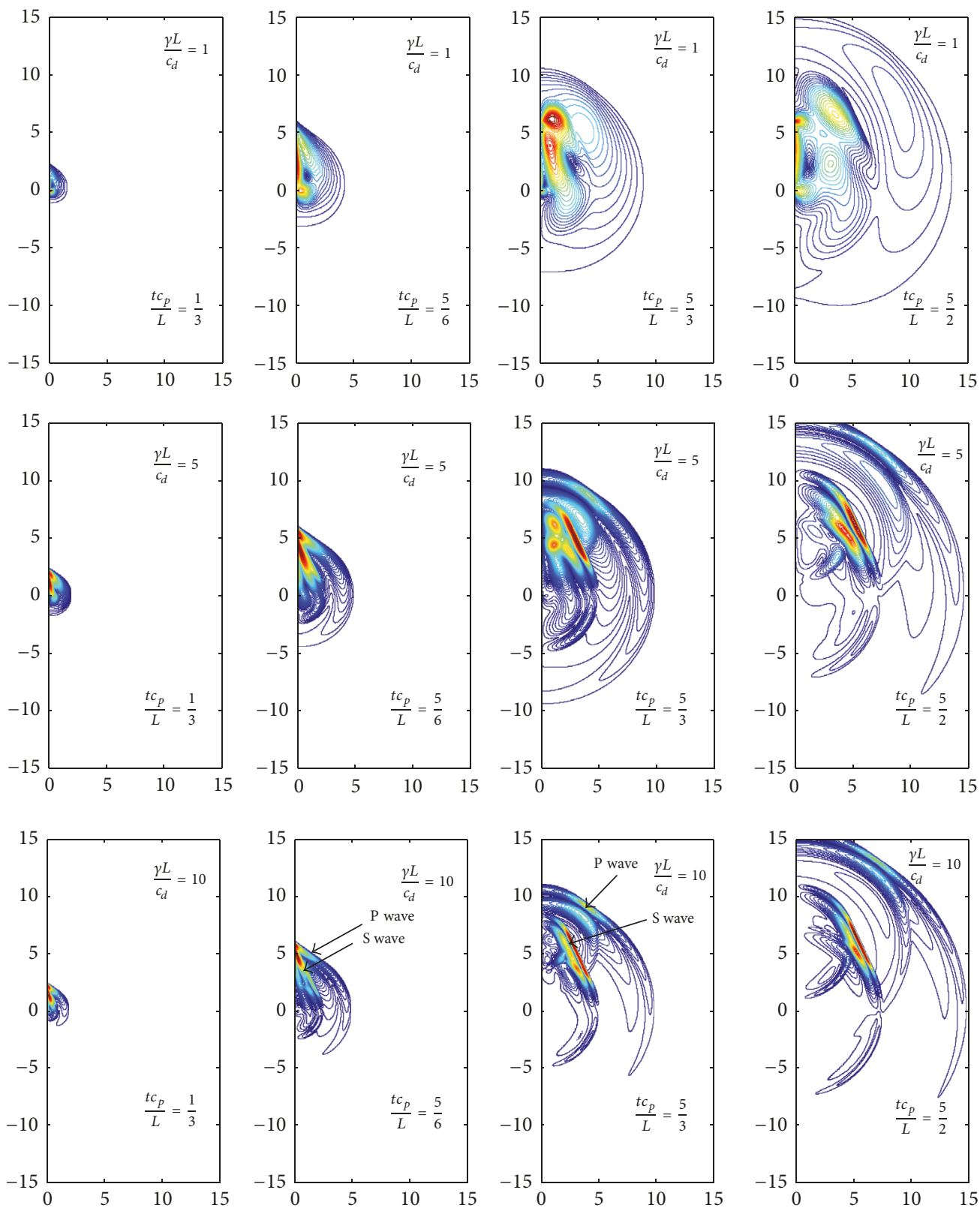

FIGURE 7: Wave fronts as a function of time and the spatial coordinates. The values of the parameters used are $c_{p}=5000 \mathrm{~m} / \mathrm{s}, c_{s}=3000 \mathrm{~m} / \mathrm{s}$, $L=6 \mathrm{~m}$, and three different values of the parameter $\gamma$.

$$
\begin{aligned}
& u_{\phi}(r, z, t) \sim p_{0} \gamma^{2} \frac{F_{2}(\phi)}{R} e^{-\gamma\left(t-R / c_{s}+L\left(\cos \phi / c_{s}-1 / c_{d}\right)\right)} \\
& \cdot \frac{1-e^{\gamma L\left(\cos \phi / c_{s}-1 / c_{d}\right)}}{1-e^{\gamma \Delta L\left(\cos \phi / c_{s}-1 / c_{d}\right)}} .
\end{aligned}
$$

In the limit $\Delta L \rightarrow 0$ and $\gamma L / c_{d} \ll 1$, the following expression is obtained:

$$
\begin{gathered}
u_{R}(r, z, t)=\frac{L a^{2}}{2 \mu c_{p} R}\left(1-2\left(\frac{c_{s}}{c_{p}}\right)^{2} \cos ^{2}(\phi)\right) \\
.\left[1-\frac{\gamma L}{2 c_{p}}\left(\cos \phi-\frac{c_{p}}{c_{d}}\right)\right] \frac{d^{2}}{d t^{2}}\left\{p\left(t-\frac{R}{c_{p}}\right)\right\}
\end{gathered}
$$

$$
\begin{aligned}
& u_{\phi}(r, z, t)=\frac{L a^{2}}{2 \mu c_{s} R} \sin (2 \phi) \\
& \cdot\left[1-\frac{\gamma L}{2 c_{s}}\left(\cos \phi-\frac{c_{s}}{c_{d}}\right)\right] \frac{d^{2}}{d t^{2}}\left\{p\left(t-\frac{R}{c_{s}}\right)\right\} .
\end{aligned}
$$

Figure 9 shows the angular distribution of $\mathrm{P}$ and $\mathrm{S}$ waves in the case of finite velocity of detonation VOD obtained from (22), where dashed line represented Heelan's solution and continuous line represented the case of VOD finite.

The velocity field obtained from (22) is identical to that of Heelan, except for the geometric factor: $1-\left(\gamma L / 2 c_{p, s}\right)(\cos \phi-$ 


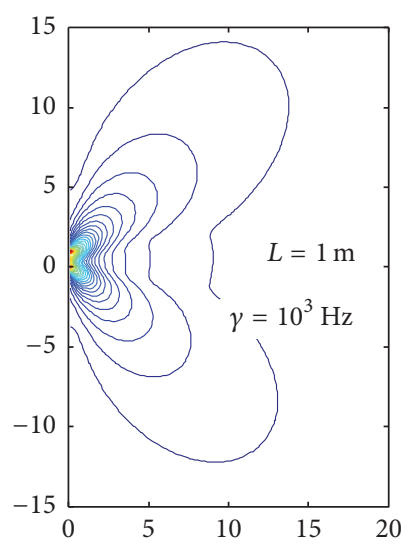

(a)

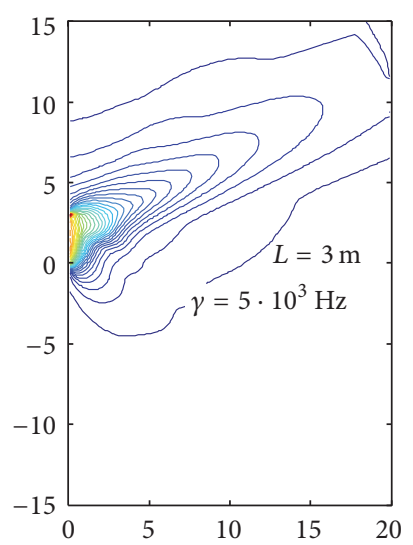

(e)

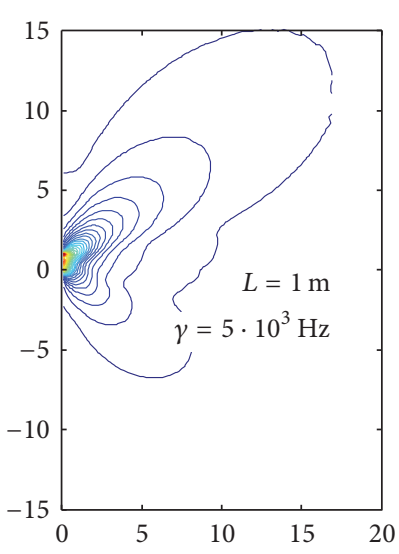

(b)

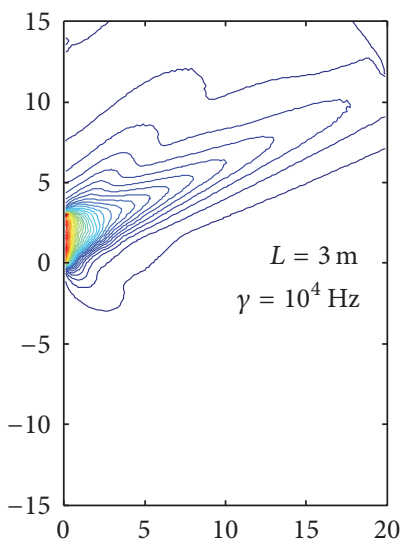

(f)

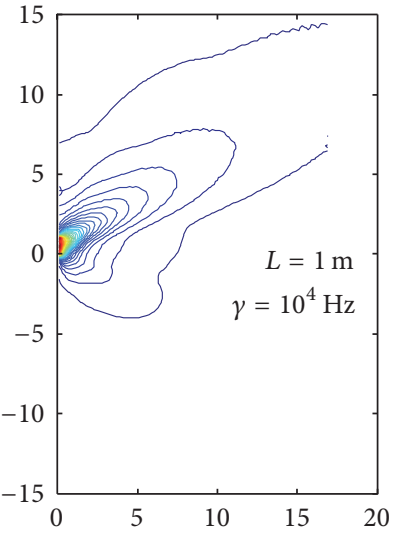

(c)

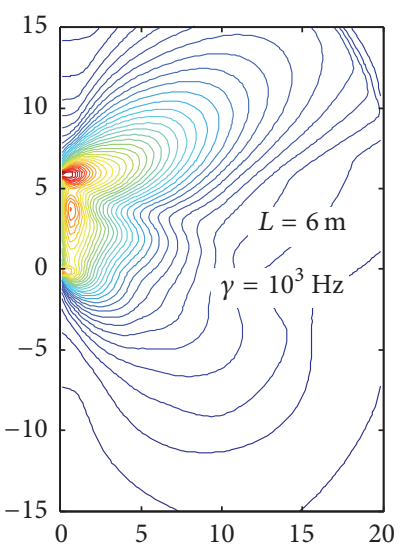

(g)

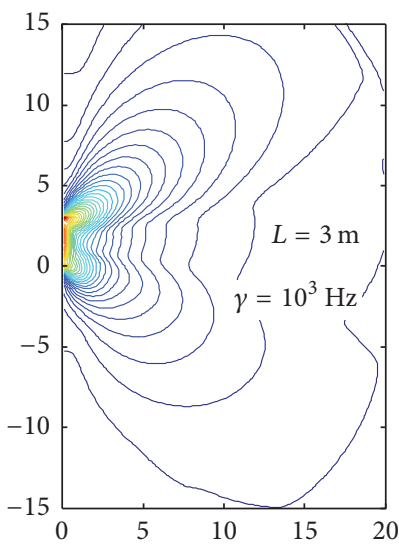

(d)

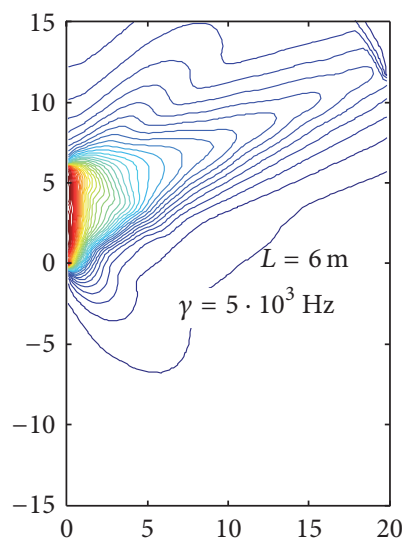

(h)

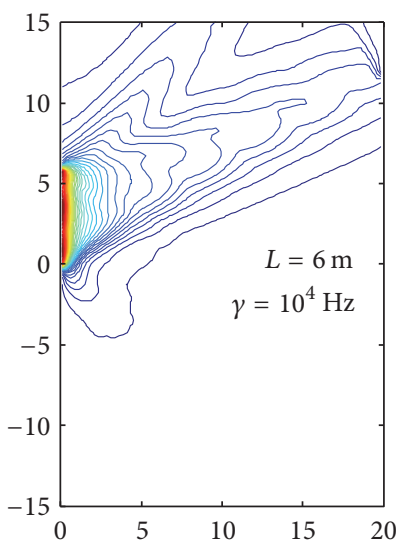

(i)

Figure 8: PPV diagrams numerically obtained from (17).

$\left.c_{p, s} / c_{d}\right)$. This geometric factor represents a first correction to Heelan's solution in the case of finite length of the explosive and finite VOD. In the case $\gamma L / c_{p, s} \rightarrow 0$, the velocity field is reduced to the solution of Heelan.

\section{Conclusions}

This paper demonstrates that the seismic radiation in the far field produced by an explosive column of finite length can be modeled by overlapping of asymptotic solutions in the far field of vibration (Heelan's solution); the results agree quite well with exact solutions $[12,13]$. The asymptotic solutions found in this work allow reducing the calculation time and facilitating the understanding of the phenomena of vibration in the elastic medium. In addition, it is shown that the geometry and structure of the peak particle velocity (PPV) diagrams depend strongly on the parameters $\gamma L / c_{p, s}$ and $c_{p, s} / c_{d}$. The asymptotic model developed in this paper can be 


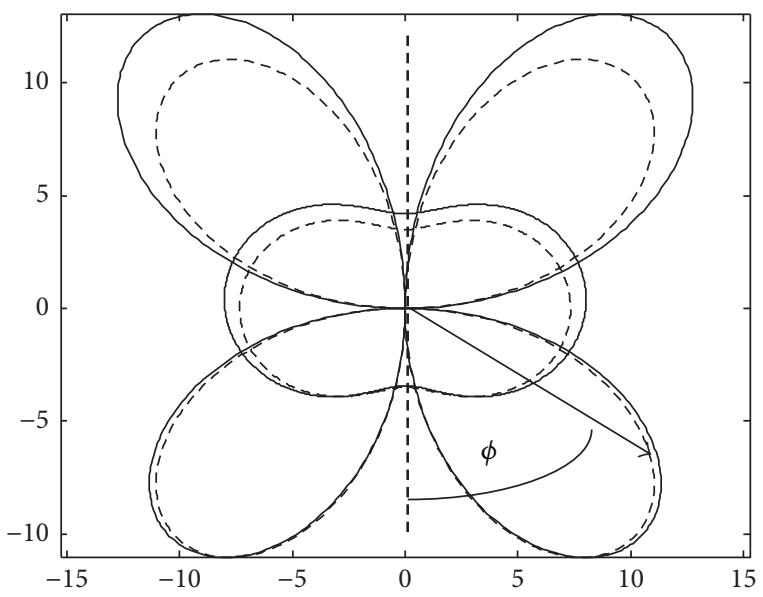

FIgURE 9: Angular distribution of $\mathrm{P}$ and $\mathrm{S}$ waves in the case of finite velocity of detonation VOD obtained from (22) (filled line).

extended to more complex problems, such as the interaction between two or more columns of explosives.

\section{Conflicts of Interest}

The authors declare that they have no conflicts of interest.

\section{References}

[1] P. A. Heelan, "On the theory of head waves," Geophysics, vol. 18, pp. 871-893, 1953.

[2] P. A. Heelan, "Radiation from a cylindrical source of finite length," Geophysics, vol. 18, pp. 685-696, 1953.

[3] D. W. Jordan, "The stress wave from a finite cylindrical explosive source," Journal of Mathematics and Mechanics, vol. 11, pp. 503$551,1962$.

[4] P. Hazebroek, "Elastic waves from a finite line source," Proceedings of the Royal Society A Mathematical, Physical and Engineering Sciences, vol. 294, no. 1436, pp. 38-65, 1966.

[5] A. M. Abo-Zena, "Radiation from a finite cylindrical explosive source," Geophysics, vol. 42, no. 7, pp. 1384-1393, 1977.

[6] J. A. Meredith, Numerical and analytical modeling of downhole seismic sources: the near and far field, Massachusetts Institute of Technology, 1990.

[7] J. A. Meredith, C. H. Cheng, and M. N. Toksoz, Modelling of downhole seismic sources $i$ : an analysis of the heelan/brekhovskikh results and comparison of point source radiation to radiation from boreholes, Massachusetts Institute of Technology. Earth Resources Laboratory, 1990.

[8] J. A. Meredith, M. N. Toksöz, and C. H. Cheng, "Secondary shear waves from source boreholes," Geophysical Prospecting, vol. 41, no. 3, pp. 287-312, 1993.

[9] K. M. Tubman, Full waveform acoustic logs in radially layered boreholes, Massachusetts Institute of Technology, 1984.

[10] K. M. Tubman, C. H. Cheng, and M. N. Toksöz, "Synthetic full waveform acoustic logs in cased boreholes," Geophysics, vol. 49, no. 7, pp. 1051-1059, 1984.

[11] D. P. Blair and A. Minchinton, "Near Field Blast Vibration Models," in Proceedings of The 8th International Symposium on
Rock Fragmentation by Blasting-Fragblast, vol. 8, pp. 152-159, Santiago, Chile, 2006.

[12] D. P. Blair, "A comparison of Heelan and exact solutions for seismic radiation from a short cylindrical charge," Geophysics, vol. 72, no. 2, pp. E33-E41, 2007.

[13] H. P. Rossmanith and N. Kouzniak, "Supersonic detonation in rock: part 2 particle displacements and velocity fields for single and multiple non-delayed and delayed detonating blast holes," International Journal of Blasting and Fragmentation, vol. 8, pp. 95-117, 2004.

[14] D. P. Blair, "Blast vibration dependence on charge length, velocity of detonation and layered media," International Journal of Rock Mechanics and Mining Sciences, vol. 65, pp. 29-39, 2014.

[15] L. Triviño, B. Mohanty, and A. Munjiza, "Investigation of seismic radiation patterns from cylindrical explosive charges by analytical and combined finite-discrete element methods," in Proceedings of The 9th International Symposium On Rock Fragmentation by Blasting, pp. 415-426, Granada, Spain, 2009.

[16] R. Kumar, D. Choudhury, and K. Bhargava, "Determination of blast-induced ground vibration equations for rocks using mechanical and geological properties," Journal of Rock Mechanics and Geotechnical Engineering, vol. 8, no. 3, pp. 341-349, 2016.

[17] M. W. Lee and A. H. Balch, "Theoretical seismic wave radiation from a fluid-filled borehole," Geophysics, vol. 47, no. 9, pp. 13081314, 1982.

[18] C. Yi, D. Johansson, U. Nyberg, and A. Beyglou, "Stress wave interaction between two adjacent blast holes," Rock Mechanics and Rock Engineering, vol. 49, no. 5, pp. 1803-1812, 2016.

[19] L. F. Triviño, B. Mohanty, and B. Milkereit, "Seismic waveforms from explosive sources located in boreholes and initiated in different directions," Journal of Applied Geophysics, vol. 87, pp. 81-93, 2012.

[20] S.-H. Chen, J. Wu, and Z.-H. Zhang, "Influence of millisecond time, charge length and detonation velocity on blasting vibration," Journal of Central South University, vol. 22, no. 12, pp. 4787-4796, 2015.

[21] D. E. Grady, M. E. Kipp, and C. S. Smith, "Explosive fracture studies on oil shale," SPE Journal, vol. 20, pp. 349-356, 1980.

[22] D. B. Larson, "Explosive energy coupling in geologic materials," International Journal of Rock Mechanics and Mining Sciences, Sciences and Geomechanics Abstracts, vol. 19, pp. 157-166, 1982. 


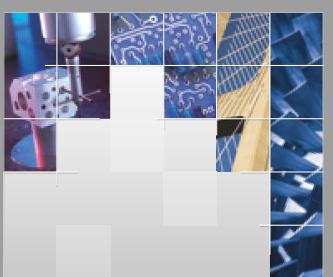

\section{Enfincering}
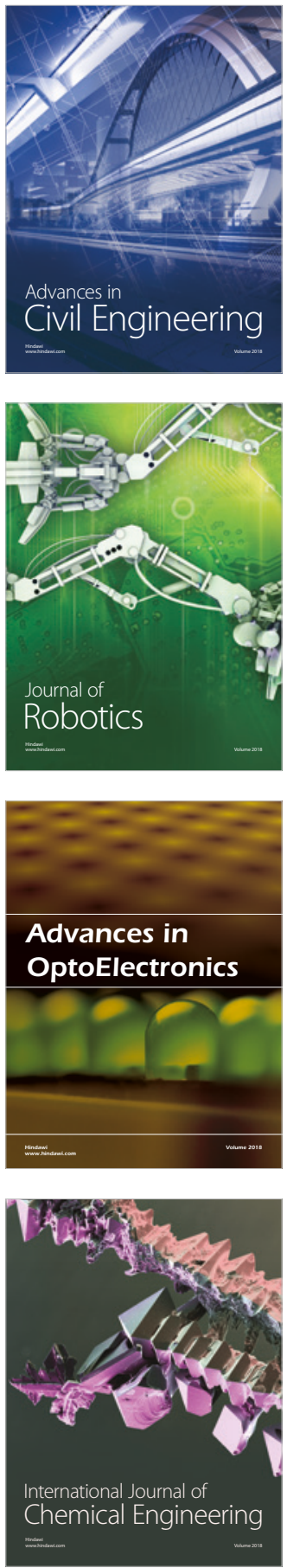

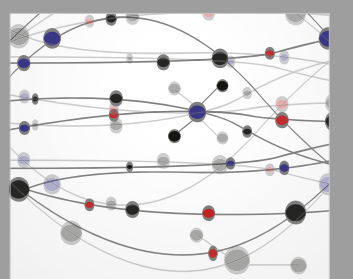

\section{Rotating \\ Machinery}

The Scientific World Journal

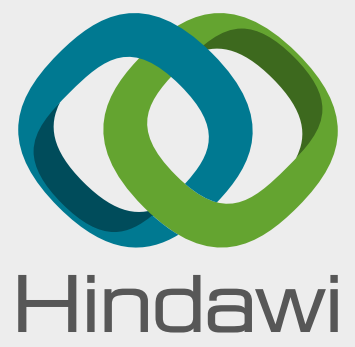

Submit your manuscripts at

www.hindawi.com
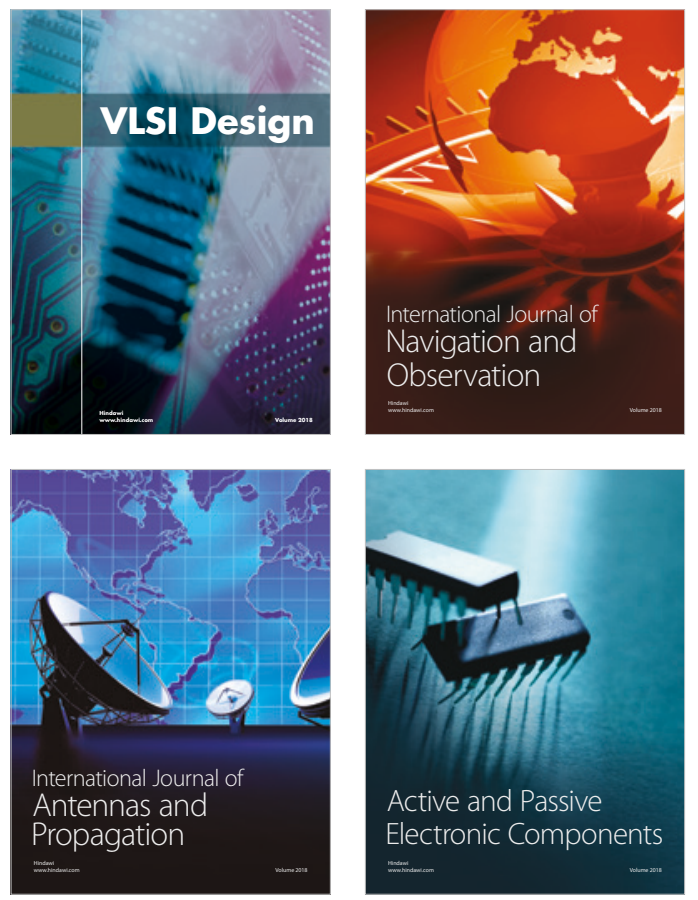
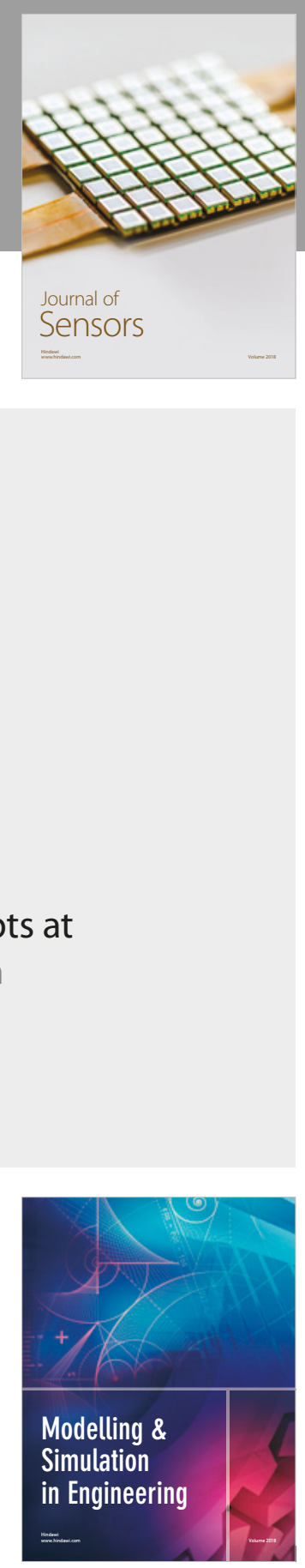

\section{Advances \\ Multimedia}
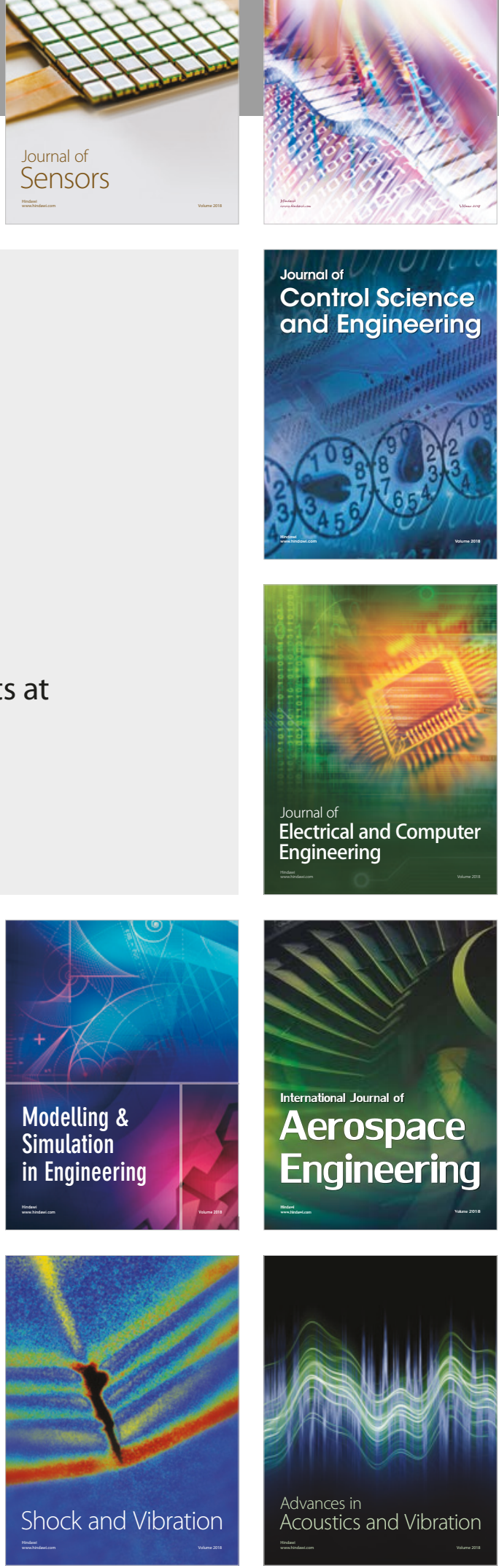